\title{
Obesity and chronic disease; the power of small group dynamics to change lifestyles
}

\section{Editorial}

It has become readily apparent to public health systems that obesity has grown into a global epidemic replacing starvation and infectious diseases as the number one disabling condition of populations throughout the world according to the World Health Organization (WHO). The high cost of obesity emerges through an array of serious and sometimes terminal chronic diseases comorbid with obesity. These diseases create intervening variable factors such as pain and disability and early mortality as well as loss of worker productivity and loss of working days among employed populations within a nation. Indeed, the chronic long-term health problems directly related to obesity are so serious; they can be used as a valid measure of the economic and political stability of any nation or region when applying standard country risk assessment (CRA) protocols. Consequently, a low CRA can result in reduced Direct Foreign Investment based on high risk assessments of poor population productivity. Further, the cost burden of delivering health care to populations suffering under high prevalence of morbid obesity is severe and debilitating to any nation's economy with subsequent downward pressure on the standard of living for the entire population. Unfortunately, many of the community and nation-wide public health education programs have been only marginally successful in creating a long term change in individual, family, and community positive healthy lifestyle decisions for national populations globally. The new effort may increasingly focus on small group settings for the presentation of healthy lifestyle education while relying on mutual support and encouragement among group members to adopt healthy lifestyles and maintain that decision in the long term with the expectation of experiencing significant improvements in the health status of participants.

Public health policy and education programs globally need to focus on sponsoring many small group health programs interspersed throughout national populations in every nation and region. As the overall health of program participants begin to offer personal testimonies of the effectiveness of small group dynamics in intervening positively in adverse health outcomes, it could be expected that the prevailing model of small group success will create incentives for more small groups to function in virtually every community within nations. Some small group models may only meet weekly for about two to three hours each week; but, through modern social media contact, group members can maintain daily communication with other members of the group and the group leader; thus, maintaining group member participation in the healthy lifestyle choices being taught and discussed in the group meetings.

The expected personal and social impact could be enormous as group members lose weight and experience increased energy levels and reduced levels of pain and suffering from chronic diseases
Volume 5 Issue 4 - 2017

\author{
Michael W Popejoy \\ Florida International University, USA
}

Correspondence: Michael W Popejoy, Florida International University, USA, Email dr_popejoy@hotmail.com

Received: March 27, 2017 | Published: March 31, 2017

related to obesity. The economic impact for each nation could be equally enormous as employee sick days are reduced and employee productivity at work is increased as a result of better health and greater levels of energy, enthusiasm, and personal and family satisfaction and happiness. At least initially, the benefits may be difficult to accurately measure; however, as progress continues along the path of improved health for all participants, then it is obvious that measurable benefits can be recorded and reported. As these reports gain overall recognition both within and between nations and regions, more healthy lifestyle groups will form resulting in even further positive benefits to population health. The medical community and public health agencies and the government within each nation globally should begin to initiate a national plan to begin forming small community health education support groups wherever possible within national borders. Over time, it will become apparent that significant population health improvements will be observable and sustainable offering obvious economic benefits to each nation. It is critical in this WHO declared epidemic of obesity; and, the chronic comorbid disease burden faced by all nations that such programs must be implemented immediately and sustained by whatever methods are at the disposal of local, regional, and national governments. The public health problem is a public administration burden and it falls to governments to protect the health of its populations. There is no time to lose as people everywhere are suffering and dying from obesity related chronic diseases.

\section{Acknowledgements}

None.

\section{Conflict of interest}

Author declares that there is no conflict of interest. 